\title{
STEADY-STATE CRACK GROWTH AND WORK OF FRACTURE FOR SOLIDS CHARACTERIZED BY STRAIN GRADIENT PLASTICITY
}

\author{
YUEGUANG WEI and JOHN W. HUTCHINSON \\ Division of Engineering and Applied Sciences, Harvard University, Cambridge, MA 02138, U.S.A.
}

(Received 10 January 1997; in revised form 11 March 1997)

\begin{abstract}
Mode I steady-state crack growth is analysed under plane strain conditions in small scale yielding. The elastic-plastic solid is characterized by a generalization of $\mathbf{J}_{2}$ flow theory which accounts for the influence of the gradients of plastic strains on hardening. The constitutive model involves one new parameter, a material length $l$, specifying the scale of nonuniform deformation at which hardening elevation owing to strain gradients becomes important. Gradients of plastic strain at a sharp crack tip result in a substantial increase in tractions ahead of the tip. This has important consequences for crack growth in materials that fail by decohesion or cleavage at the atomic scale. The new constitutive law is used in conjunction with a model which represents the fracture process by an embedded traction-separation relation applied on the plane ahead of the crack tip. The ratio of the macroscopic work of fracture to the work of the fracture process is calculated as a function of the parameters characterizing the fracture process and the solid, with particular emphasis on the role of $l$. (C) 1997 Elsevier Science Ltd
\end{abstract}

Keywords: A. crack tip plasticity, B. elastic-plastic material, A. fracture, A. fracture toughness.

\section{INTRODUCTION}

The first part of this paper addresses the role of plastic strain gradients in elevating the tractions ahead of the tip of a sharp crack advancing under steady-state conditions. An extension of incremental plasticity theory which incorporates a dependence of hardening on both plastic strains and strain gradients is employed to characterize the solid. A new constitutive length parameter, $l$, is added to the standard set of parameters characterizing the elastic-plastic solid : the Young's modulus, E, Poisson's ratio, v, tensile yield stress, $\sigma_{\mathrm{Y}}$, and strain hardening index, $N$. Gradient effects become important when the scale of nonuniform straining becomes of order $l$. The problem solved in Section 4 is for small scale yielding. The traction distribution near the crack tip is obtained in terms of the remotely applied intensity field. No near-tip fracture criterion is imposed on the solution.

By contrast, the second part of the paper, in Section 5, makes use of the strain gradient constitutive relation in a fracture model that embeds a prescribed tractionseparation behavior on the plane ahead of the crack tip to simulate the fracture process. Computations based on the embedded fracture process zone model (the EPZ model) provide the relationship between the steady-state macroscopic work fracture 
and the work of the fracture process. The focus of this part of the study is the interplay between the new constitutive length parameter $l$ and the maximum separation traction required by the fracture process.

Motivation for exploring the effect of plastic strain gradients on crack tip tractions derives from deficiencies based on conventional plasticity theory, coupled with recent developments in the strain gradient theory suggesting that more realistic predictions may be enabled by the theory. The deficiencies concern the maximum stress levels attainable ahead of a growing sharp crack surrounded by a plastic zone. According to results from conventional plasticity for growing plane strain cracks in mode $I$, the maximum normal stress that can be attained ahead of the tip is about 2.6 times the initial tensile yield stress $\sigma_{\mathrm{Y}}$, if the material is elastic-perfectly plastic (Drugan et al., 1982). Strain hardening gives rise to a stress singularity, but numerical studies for growing cracks in conventional elastic-plastic solids indicate that this singularity must be exceptionally weak. For relevant distances ahead of the tip, the maximum normal stress never exceeds about 4 , or at most 5 , times $\sigma_{\mathrm{Y}}$, depending on $N$. Maximum crack tip tractions this low present a "paradox" for fracture occurring by cleavage or decohesion at the atomic scale (Bagchi and Evans, 1996). Atomic separation requires traction levels on the order of the theoretical lattice strength (roughly $E / 30$ ), which for most metals is more typically on the order of $10 \sigma_{\mathrm{Y}}$ or more. Thus, conventional plasticity theory predictions would appear to rule out a fracture mechanism based on atomic separation whenever a well developed plastic zone surrounds the crack tip. The "paradox" pertains as well to crack propagation along strong metal-ceramic interfaces where similarly low normal interface tractions within the plastic zone are predicted.

Several proposals have been put forward to justify higher crack tip stresses. When the crack velocity is sufficiently high and material rate effects are taken into account, a robust elastic singularity within the plastic zone re-emerges, and traction levels required for lattice separation arise (Freund and Hutchinson, 1985). This description only applies to cracks running at high speeds. It does not explain how cracks are able to propagate quasi-statically when atomic separation is the fracture mechanism. Recent experiments on metal-ceramic interfaces (Elssner et al., 1994; Bagchi et al., 1994; Bagchi and Evans, 1996) have provided detailed evidence of quasi-static propagation of interface decohesion cracks in the presence of substantial plasticity in the metal. Another approach, pursued most recently by Suo et al. (1993) and Beltz, et al. (1996), employs a model which excludes plasticity within some distance $D$ from the crack tip. In this way, an clastic stress singularity exists at the tip, enabling the normal traction to attain levels necessary for lattice separation at distances on the order of an atomic spacing from the tip. A difficulty with this type of model, which will be more evident shortly, is the retention of conventional plasticity to describe material deformation outside the elastic exclusionary zone. We will argue that a consistent application of this class of model may require the use of an enhanced plasticity model such as the strain gradient plasticity employed in the present study.

Experimental evidence for elevated flow stresses due to nonuniform plastic straining at small length scales has come from several sources. Indentation tests (Stelmashenko et al., 1993; Ma and Clarke, 1995; Poole et al., 1996) of several metals have revealed that elevations in measured hardening set in when the size of the indentation is smaller 
than about $10-20 \mu \mathrm{m}$, and elevations by factors of 2 or 3 have been observed for indentations as small as about $1 \mu \mathrm{m}$. Torsion tests on fine copper wires (Fleck et al., 1994) similarly reveal factors of 3 in elevation of effective flow stress for the thinnest wires tested (12 $\mu \mathrm{m}$ diameter) compared to the thickest tested (120 $\mu \mathrm{m}$ diameter) at corresponding strains. The material length parameter, $l$, which will be defined precisely in the next section, was inferred to be about $4 \mu \mathrm{m}$ based on the wire torsion data for copper. The physical basis of strain gradient plasticity (Fleck et al., 1994) derives from geometrically necessary dislocations generated by gradients in plastic strain. When the gradient of strain become large enough compared to the strain, the density of geometrically necessary dislocations becomes comparable to the density of dislocations generated by the strain itself (referred to as statistically stored dislocations). It is then that the effect on flow stress of the gradient in plastic strain becomes important. Given the significance of flow stress elevation in both wire torsion and indentation at scales below about $10-20 \mu \mathrm{m}$, it seems highly plausible that similar effects should prevail at the same scale at the crack tip. It is this reasoning that underlies the present study.

The first problem to be addressed is depicted in Fig. 1. It shows a semi-infinite crack propagating in steady-state under small scale yielding in plane strain conditions in a homogeneous elastic - plastic solid. The strain gradient theory of plasticity used to characterize the solid is specified in the next section. The remote field, which is unaffected by gradient effects, is prescribed to be the classical elastic mode I solution with an $r^{-1 / 2}$ dependence and the stress intensity factor $K$ as amplitude. An active plastic zone surrounds the tip, followed by a wake of elastic unloading with residual plastic strain and stress. A useful reference estimate of the half-height of the plastic zone is

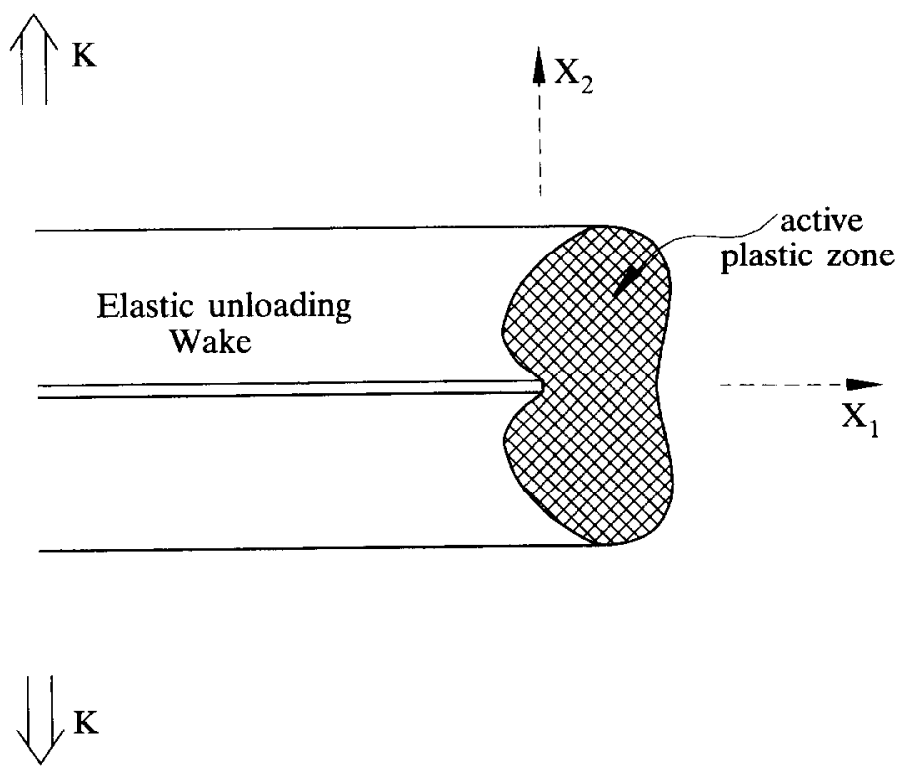

Fig. 1. Steady-state crack growth in small scale yielding. 


$$
R_{\mathrm{P}}=\frac{1}{3 \pi} \frac{K^{2}}{\sigma_{\mathrm{Y}}^{2}}
$$

Apart from the new material constitutive parameter $l, R_{\mathrm{P}}$, or some multiple of it, is the only length quantity in the first problem. Dimensional considerations dictate that the form of the solution, for the stresses, for example, is

$$
\sigma_{i j}=\sigma_{\mathrm{Y}} \bar{\sigma}_{i j}\left(\frac{\mathbf{x}}{R_{\mathrm{P}}}, \frac{l}{R_{\mathrm{P}}}, N, \frac{\sigma_{\mathrm{Y}}}{E}, v\right),
$$

where the barred quantities are dimensionless functions of the arguments listed. The aim of the first part of the paper is to reveal the dependence on $l / R_{\mathrm{p}}$, with primary emphasis on the tractions on the plane ahead of the tip.

The present study appears to be the first to investigate plastic strain gradient effects on growing cracks and fracture toughness. Studies of stationary cracks within the context of strain gradient plasticity have shown a significant dependence of the neartip stress distribution on the mode of loading and the class of strain gradient solids. The simplest class of solids, designated as couple stress (CS) solids in the next section, depend only on strain gradients which derive from rotation gradients (deformation curvatures), and this class falls within the category of couple stress theory. Stationary mode III cracks (Schiermeier et al., 1996) in the CS solid display a substantial elevation of the shear traction ahead of the tip, as compared to the tractions predicted for the conventional plastic solid. By contrast, stationary mode I cracks (Xia and Hutchinson, 1996; Huang et al., 1997) in the CS solid hardly experience any elevation in normal traction ahead of the crack tip. The behavior difference between these two modes arises because the asymptotic near-tip field for the mode I problem in the CS solid turns out to be irrotational. Rotation gradients vanish to lowest order, thereby producing no appreciable hardening or traction elevation. On the other hand, rotation gradients are inescapable in the mode III problem, significantly altering near-tip tractions. When both stretch and rotation gradients are incorporated in the constitutive response of the solid (labeled the SG solid in the next section), significant traction elevation near the tip due to strain gradient hardening occurs as well for the stationary mode I crack (Schiermeier and Hutchinson, 1996). The SG solid will be used in this paper in the study of steady-state crack growth and in conjunction with the EPZ model of steady-state fracture toughness.

\section{CONSTITUTIVE RELATION : STRAIN GRADIENT PLASTICITY}

The study is carried out within the context of the small strain framework. The version of strain gradient plasticity proposed by Fleck and Hutchinson (1997) is used, and this section draws heavily from that reference. Following those authors, a deformation version of the theory is specified first. This is a small strain, nonlinear elasticity which incorporates an isotropic dependence on the strain gradients. This version is then rendered to incremental form by identifying a yield surface and postulating normality of plastic flow. The result is a generalization of the widely used 
$\mathbf{J}_{2}$ flow theory of plasticity. When strain gradients are sufficiently small, the constitutive relation reduces to $J_{2}$ flow theory. Thus, for example, the plasticity in the outer part of the plastic zone will be only slightly affected by gradient effects. The material becomes fully characterized when the uniaxial tensile stress-strain behavior and the material length parameter $l$ are specified. The constitutive law, as posed, expresses increments of strains and strain gradients in terms of increments of the stress quantities. For almost any problem whose solution employs a displacement-based finite element method, such as the present solution method for the crack problems, it is essential that the constitutive relation be inverted analytically. Numerical inversion would be far too inefficient. Thus, a major objective of this section is to present the inverted form of the incremental constitutive law.

\subsection{Deformation theory}

With $u_{i}$ as the displacements, $\varepsilon_{i j}=\frac{1}{2}\left(u_{i, j}+u_{j, i}\right)$ is the strain tensor and $\eta_{i j k}=\eta_{j i k}=u_{k, i j}$ is the tensor of second gradient of displacements. The latter can be expressed in terms of the gradients of strains as $\eta_{i j k}=\varepsilon_{j k, i}+\varepsilon_{i k, j}-\varepsilon_{i j, k}$. An incompressible solid has $\varepsilon_{k k}=0$ and $\eta_{i k k}=0$. Let $\varepsilon_{i j}^{\prime}=\varepsilon_{i j}-\frac{1}{3} \delta_{i j} \varepsilon_{\mathrm{pp}}$ be the strain deviator. Let $\eta_{i j k}^{\mathrm{H}}=\frac{1}{4}\left(\delta_{i k} \eta_{j \mathrm{pp}}+\delta_{j k} \eta_{i \mathrm{pp}}\right)$ be the "hydrostatic" part of $\eta$, which vanishes for incompressible deformations, and let $\eta^{\prime}=\eta-\eta^{\mathrm{H}}$ be the deviator. Consider the invariant $E$ of the strains and strain gradients defined by

$$
E^{2}=\frac{2}{3} \varepsilon_{i j}^{\prime} \varepsilon_{i j}^{\prime}+c_{1} \eta_{i i k}^{\prime} \eta_{i j k}^{\prime}+c_{2} \eta_{i j k}^{\prime} \eta_{i j k}^{\prime}+c_{3} \eta_{i j k}^{\prime} \eta_{k j i}^{\prime}
$$

Under uniform strain, $E$ is the von Mises strain invariant. The coefficients $c_{i}$ have the dimension of length ${ }^{2}$. For incompressible deformations of isotropic, centro-symmetric solids (2.1) is the general combination of quadratic invariants of the strains and strain gradients (Toupin 1962; Mindlin, 1964).

The deformation theory is based on the measure $E$. Through this measure, the strain gradients as well as the strains contribute to the energy of deformation and, therefore, to the level of stress. Following Fleck and Hutchinson (1997), this measure is expressed in two other ways. First, introduce the Smyshlyaev-Fleck (1996) orthogonal decomposition of the strain gradient tensor:

$$
\eta_{i j k}=\sum_{I=1}^{4} \eta_{i j k}^{(l)} \quad \text { with } \eta_{i j k}^{(\eta)} \eta_{i j k}^{(\eta)}=0 \text { if } I \neq J,
$$

where $\eta^{(4)} \equiv \eta^{\mathbf{H}}$ and, here, $\eta^{(I)}$ is used to replace the notation $\eta^{(I)}$ (for $I=1,3$ ) employed in the earlier references. The decomposition is given in the Appendix. Expression (2.1) can be recast as

$$
E^{2}=\frac{2}{3} \varepsilon_{i j}^{\prime} \varepsilon_{i j}^{\prime}+l_{1}^{2} \eta_{i j k}^{(1)} \eta_{i j k}^{(1)}+l_{2}^{2} \eta_{i j k}^{(2)} \eta_{i j k}^{(2)}+l_{3}^{2} \eta_{i j k}^{(3)} \eta_{i j k}^{(3)},
$$

where the length parameters $l_{i}$ are related to the $c_{i}$ 's by

$$
l_{1}^{2}=c_{2}+c_{3}, \quad l_{2}^{2}=c_{2}-\frac{1}{2} c_{3}, \quad l_{3}^{2}=\frac{5}{2} c_{1}+c_{2}-\frac{1}{4} c_{3} .
$$

In the form (2.3), it is obvious that the measure is positive definite if all the $l_{i}$ are nonzero. To obtain the third form, it is noted that for incompressible deformations the 
last two invariants in (2.3) can be expressed in terms of the invariants of the gradient of the rotation (Fleck and Hutchinson, 1997). With rotation as $\theta_{i}=\frac{1}{2} e_{i j k} u_{k_{j}}, e_{i j k}$ as the permutation symbol, and $\chi_{i j}^{\prime}=\theta_{i, j}=e_{i \mathrm{pk}} \varepsilon_{k j, \mathrm{p}}^{\prime}$ as the rotation gradients,

$$
\eta_{i j k}^{\prime(2)} \eta_{i j k}^{\prime(2)}=\frac{4}{3} \chi_{i j}^{\prime} \chi_{i j}^{\prime}+\frac{4}{3} \chi_{i j}^{\prime} \chi_{j i}^{\prime} \quad \text { and } \quad \eta_{i j k}^{\prime(3)} \eta_{i j k}^{\prime(3)}=\frac{8}{5} \chi_{i j}^{\prime} \chi_{i j}^{\prime}-\frac{8}{5} \chi_{i j}^{\prime} \chi_{j l}^{\prime}
$$

Thus, an equivalent alternative expression to (2.1) or (2.3) is

$$
E^{2}=\frac{2}{3} \varepsilon_{i j}^{\prime} \varepsilon_{i j}^{\prime}+l_{1}^{2} \eta_{i j k}^{(1)} \eta_{i j k}^{(1)}+\left(\frac{4}{3} l_{2}^{2}+\frac{8}{5} l_{3}^{2}\right) \chi_{i j}^{\prime} \chi_{i j}^{\prime}+\left(\frac{4}{3} l_{2}^{2}-\frac{8}{5} l_{3}^{2}\right) \chi_{i j}^{\prime} \chi_{j i}^{\prime}
$$

The following two combinations of the length parameters were designated as being a couple stress solid (CS) and a strain gradient solid (SG) by Fleck and Hutchinson (1997) :

$$
\begin{aligned}
& \text { CS: } l_{1}=0, \quad l_{2}={ }_{2}^{1} l, \quad l_{3}=\sqrt{5}{ }_{24} l, \\
& \mathrm{SG}: \quad l_{1}=l, \quad l_{2}=\frac{1}{2} l, \quad l_{3}=\sqrt{\frac{5}{24}} l \text {. }
\end{aligned}
$$

These two particular combinations were discussed within the context of stationary crack problems in Section 1. The SG solid will be used in the present study. It should be emphasized that the relative weight of the second term in (2.6), which incorporates the stretch gradients, to the third term involving only rotation gradients remains to be tied down by an appropriate set of experiments. The formulation for the CS solid can be framed within a restricted class of theories (couple stress theory), which depends only on the components of $\eta$ due to rotation gradients.

Let $\sigma_{i j}$ be the symmetric second order stress tensor and $\tau_{i j k}=\tau_{j i k}$ the higher order stress tensor such that the work increment per unit volume associated with an arbitrary variation of the displacements is

$$
\delta W=\sigma_{i j} \delta \varepsilon_{i j}+\tau_{i j k} \delta \eta_{i j k} .
$$

With $W(\varepsilon, \eta)$ as the energy density function,

$$
\sigma_{i j}=\frac{\partial W}{\partial \varepsilon_{i j}} \text { and } \tau_{i j k}=\frac{\partial W}{\partial \eta_{i j k}} .
$$

In the strain gradient deformation theory that generalizes $\mathrm{J}_{2}$ deformation theory, $W$ is taken to be a function of $E$, together with contributions from $\varepsilon_{j j}^{2}$ and $\eta_{i j k}^{\mathrm{H}} \eta_{i j k}^{\mathrm{H}}$ to account for compressibility. The functional dependence is chosen to reproduce a specified uniaxial tensile stress-strain curve.

The principle of virtual work reads

$$
\int_{V}\left[\sigma_{i j} \delta \varepsilon_{i j}+\tau_{i j k} \delta \eta_{i j k}\right] \mathrm{d} V=\int_{V} f_{i} \delta u_{i} \mathrm{~d} V+\int_{S} t_{i} \delta u_{i} \mathrm{~d} S+\int_{S} r_{i}\left(D \delta u_{i}\right) \mathrm{d} S,
$$

where $\mathrm{d} V$ and $\mathrm{d} S$ are the volume and surface area elements, $f_{i}$ is the body force per unit volume, $t_{i}$ is the surface traction and $r_{i}$ is the double stress traction. At the surface, the gradient of any quantity $y$ is decomposed into tangential and normal components according to

$$
y_{, i}=D_{i} y+n_{i} D y \quad \text { with } \quad D() \equiv n_{j}()_{, j} \quad \text { and } \quad D_{i}() \equiv\left(\delta_{i j}-n_{i} n_{j}\right)()_{, j} .
$$


Equilibrium equations and relations between the tractions and the stress quantities which follow from the principle are

$$
\begin{gathered}
\sigma_{i k, i}-\tau_{i j k, i j}=-f_{k}, \\
t_{k}=n_{i}\left(\sigma_{i k}-\tau_{i j k, j}\right)+n_{i} n_{j} \tau_{i j k}\left(D_{\mathrm{p}} n_{\mathrm{p}}\right)-D_{j}\left(n_{i} \tau_{i j k}\right) \quad \text { and } \quad r_{k}=n_{i} n_{j} \tau_{i j k} .
\end{gathered}
$$

Line loads along surface edges are omitted here (cf. Fleck and Hutchinson, 1997).

\subsection{Flow theory}

The incremental, or flow, theory which follows (Fleck and Hutchinson, 1997) has the property that it coincides with the deformation theory specified above for restricted classes of stress histories corresponding to proportional loading. This flow theory satisfies the fundamental requirements of stability and convexity, as shown in the reference just cited.

Let $\sigma_{i j}^{\prime}=\sigma_{i j}-\frac{1}{3} \delta_{i j} \sigma_{\mathrm{pp}}$ be the stress deviator. Let $\tau_{i j k}^{(4)} \equiv \tau_{i j k}^{\mathrm{H}}=\frac{1}{4}\left(\delta_{i k} \tau_{j \mathrm{pp}}+\delta_{j k} \tau_{\mathrm{ppp}}\right)$ be the "hydrostatic" part of $\tau$, with $\tau^{\prime}=\tau-\tau^{\mathrm{H}}$ as the deviator. Decompose $\tau$ into the four mutually orthogonal tensors $\tau^{(n)}$ as in (2.2).

The elastic strain energy density of the solid is taken to be

$$
W^{\mathrm{e}}=E\left[\frac{1}{2(1+v)} \varepsilon_{i j} \varepsilon_{i j}+\frac{v}{2(1+v)(1-2 v)} \varepsilon_{j j}^{2}\right]+E l_{c}^{2}\left[\sum_{I=1}^{4} \kappa_{\mathrm{e}}^{(I)^{2}} \eta_{i j k}^{(i)} \eta_{i j k}^{(n)}\right] .
$$

The length scales $l_{\mathrm{e}} \kappa_{\mathrm{e}}^{(I)}\left(I=1,4\right.$; where $l_{\mathrm{e}}$ has the dimension of length and the $\kappa_{\mathrm{e}}^{(I)}$ are dimensionless) characterizing the strain gradient energy contribution in the elastic range are adopted to ensure a positive definite energy density. They are not present on the basis of any physical grounds at the scales of interest for plastic strain gradient phenomena. These length scales must be chosen sufficiently small such that they have essentially no influence on any solution for which plastic strain gradients dominate elastic strain gradients. For elastic deformations, it follows from (2.9) that

where

$$
\sigma_{i j}=L_{i j k l} \varepsilon_{k i} \quad \text { and } \quad \tau_{i j k}=J_{i j k m n} \eta_{l m n}
$$

$$
L_{i j k l}=\frac{E}{2(1+v)}\left[\delta_{i j} \delta_{j l}+\delta_{i l} \delta_{j k}+\frac{2 v}{(1-2 v)} \delta_{i j} \delta_{k l}\right] \text { and } J_{i j k l m n}=2 E l_{\mathrm{e}}^{2} \sum_{I=1}^{4} \kappa_{\mathrm{e}}^{\left(I^{2}\right.} T_{i j k i m n}^{(n)} .
$$

The isotropic projection tensors $\mathbf{T}^{(f)}$ are specified in the Appendix. They share the indicial symmetries: $T_{i j k l m n}^{(n)}=T_{j i k m n}^{(l)}=T_{i j k m l n}^{(n)}=T_{l m n j k}^{(n)}$ and are defined such that for any tensor $\eta$ with indicial symmetry $\eta_{i j k}=\eta_{j i k}$ (Smyshlyaev and Fleck, 1996)

$$
T_{i, k i m n}^{(I)}=\frac{1}{2}\left(\frac{\partial \eta_{i j k}^{(i)}}{\partial \eta_{i m n}}+\frac{\partial \eta_{i j k}^{(I)}}{\partial \eta_{m i n}}\right) \quad \text { or } \eta_{i, k}^{(I)}=T_{i j k l m n}^{(I)} \eta_{l m n} \quad \text { for } I=1,4
$$

Identical connections pertain for $\tau^{(n)}$ and $\tau$. The projection tensors satisfy

$$
\begin{aligned}
T_{i j k m n}^{(I)} T_{i m n p q r}^{(I)} & =0 \quad \text { for } I \neq J, \\
& =T_{i j k p q r}^{(I)} \quad \text { for } I=J .
\end{aligned}
$$


The inverse elastic relations are

$$
\varepsilon_{i j}=M_{i j k l} \sigma_{k l} \text { and } \quad \eta_{i j k}=K_{i j k l m n} \tau_{l m n},
$$

where

$$
M_{i j k l}=\frac{1}{E}\left[\frac{1+v}{2}\left(\delta_{i k} \delta_{j l}+\delta_{i l} \delta_{j k}\right)-v \delta_{i j} \delta_{k l}\right] \text { and } \quad K_{i j k l m n}=\frac{1}{2 E l_{\mathrm{e}}^{2}} \sum_{I=1}^{4} \kappa_{\mathrm{e}}^{(I)^{-2}} T_{i j k l m n}^{(I)}
$$

An effective stress quantity $\Sigma$ generalizing the von Mises stress, $\sigma_{\mathrm{e}}=\sqrt{3 \sigma_{i j}^{\prime} \sigma_{i j}^{\prime} / 2}$, is defined in terms of the deviator stress quantities as

$$
\Sigma^{2}=\frac{3}{2} \sigma_{i j}^{\prime} \sigma_{i j}^{\prime}+l^{-2} \sum_{I=1}^{3} \kappa^{()^{-2}} \tau_{i j k}^{(n)} \tau_{i j k}^{(n)}
$$

where $l \kappa^{(I)} \equiv l_{I}(I=1,3)$ are the length quantities associated with the plastic strain gradients introduced in (2.3). Take $l$ to be the largest of the $l_{l}$, all of which are assumed to be nonzero. (The CS solid in (2.7) is a degenerate limit of the present theory. It must be considered within the more restrictive couple stress formulation (cf. Fleck and Hutchinson, 1997).) The yield surface in this generalization of $J_{2}$ flow theory is

$$
\Phi(\Sigma, Y)=\Sigma-Y,
$$

where $Y$ is the current effective tensile flow stress.

Plastic loading requires $\Sigma=Y$ and $\dot{\Sigma}>0$. Normality of plastic flow for any plastic loading increment requires

$$
\left(\dot{\varepsilon}_{i j}^{\mathrm{p}}, \dot{\eta}_{i j k}^{\mathrm{p}}\right)=\frac{\dot{\Sigma}}{h(\Sigma)}\left(\frac{\partial \Phi}{\partial \sigma_{i j}^{\prime}}, \frac{\partial \Phi}{\partial \tau_{i j k}^{\prime}}\right) .
$$

The hardening rate $h(\Sigma)$ is defined by data from a uniaxial tension test, which for the above reduces to $\dot{\varepsilon}^{\mathrm{p}}=\boldsymbol{\sigma} / h(\sigma)$. Thus, the dependence of $h$ on $\Sigma$ is identical to its dependence on $\sigma_{\mathrm{e}}$ in conventional $\mathrm{J}_{2}$ flow theory. The plastic work rate is $\sigma_{i j} \dot{\xi}_{i j}^{\mathrm{p}}+\tau_{i j k} \dot{\eta}_{i j k}^{\mathrm{p}}=\Sigma \dot{\Sigma} / h(\Sigma)$, and an effective plastic strain rate can be defined which is equal to $\dot{\Sigma} / h(\Sigma)$ (Fleck and Hutchinson, 1997). Explicit expressions for the total rates of strain and strain gradient in a plastic loading increment follow from summing elastic contributions from (2.19) and plastic contributions from (2.23). They are

$$
\begin{aligned}
\dot{\varepsilon}_{i j}= & {\left[M_{i j k l}+\frac{9}{4 h \Sigma^{2}} \sigma_{i j}^{\prime} \sigma_{k l}^{\prime}\right] \dot{\sigma}_{k l}+\frac{1}{l^{2}}\left[\frac{3}{2 h \Sigma^{2}} \sigma_{i j}^{\prime} \sum_{l=1}^{3} \kappa^{(n)^{-2}} \tau_{k l m}^{(l)}\right] \dot{t}_{k l m} } \\
\dot{\eta}_{i j k}= & \frac{1}{l^{2}}\left[\frac{3}{2 h \Sigma^{2}} \sigma_{m n}^{\prime} \sum_{l=1}^{3} \kappa^{(I)}{ }^{2} \tau_{i j k}^{(I)}\right] \dot{\sigma}_{m n}+\frac{1}{l^{4}}\left[l^{4} K_{i j k l m n}\right. \\
& \left.+\frac{1}{h \Sigma^{2}} \sum_{I=1}^{3} \kappa^{(l)^{-2}} \tau_{i j k}^{(I)} \sum_{J=1}^{3} \kappa^{(J)^{-2}} \tau_{l m n}^{(J)}\right] \dot{\tau}_{l m n}
\end{aligned}
$$


The steps for constructing the inverse to (2.24) are outlined in the Appendix. The result is

$$
\begin{aligned}
& \dot{\sigma}_{i j}=\left[L_{i j k l}-\frac{9 \mu}{(3+H) \sigma_{\mathrm{c}}^{2}} \sigma_{i j}^{\prime} \sigma_{k l}^{\prime}\right] \dot{\varepsilon}_{k l}+\left[-\frac{6 E\left(l_{\mathrm{e}} / l\right)^{2}}{(3+I I) \sigma_{\mathrm{c}}^{2}} \sigma_{i j}^{\prime} \sum_{I=1}^{3}\left(\frac{\kappa_{\mathrm{e}}^{(I)}}{\kappa^{(h)}}\right)^{2} \tau_{k l m}^{(I)}\right] \dot{\eta}_{k l m}, \\
& \dot{\tau}_{i j k}=\left[-\frac{6 E\left(l_{\mathrm{e}} / l\right)^{2}}{(3+H) \sigma_{\mathrm{e}}^{2}} \sigma_{m n}^{\prime} \sum_{l=1}^{3}\left(\frac{\kappa_{\mathrm{e}}^{(l)}}{\kappa^{(h)}}\right)^{2} \tau_{i j k}^{(n)}\right] \dot{\varepsilon}_{m n} \\
& +\left[. J_{i j k t m r n}-\frac{8(1+v) E\left(l_{\mathrm{c}} / l\right)^{4}}{(3+H) \sigma_{\mathrm{e}}^{2}} \sum_{I=1}^{3}\left(\frac{\kappa_{\mathrm{e}}^{(I)}}{\kappa^{(l)}}\right)^{2} \tau_{i j k}^{(I)} \sum_{J=1}^{3}\left(\frac{\kappa_{\mathrm{e}}^{(J)}}{\kappa^{(J)}}\right)^{2} \tau_{l m m}^{(J)}\right] \dot{\eta}_{l m n},
\end{aligned}
$$

where, as before, $\sigma_{\mathrm{e}}=\sqrt{3_{i j}^{\prime} \sigma_{i j}^{\prime} / 2}, \mu=E /[2(1+v)]$ is the shear modulus, and

$$
H=\left(\frac{\Sigma}{\sigma_{\mathrm{e}}}\right)^{2}\left[1+\frac{2 E l_{\mathrm{e}}^{2}}{h \Sigma^{2} l^{4}} \sum_{I=1}^{3} \frac{\kappa_{\mathrm{e}}^{\left(h^{2}\right.}}{\kappa^{\left(h^{4}\right.}} \tau_{i j k}^{(\eta)} \tau_{i j k}^{(\eta)}\right] \frac{h}{\mu} .
$$

The inverse does not exist in the limit $l_{\mathrm{e}} \rightarrow 0$, or when any of the $\kappa_{\mathrm{e}}^{(I)}$ are zero. The limit in which the solid is rigid in its higher order elastic moduli must be considered on its own merits, akin to the approach to rigid-plastic solids in conventional plasticity.

A piecewise-power law tensile stress-strain curve will be used to characterize the solid in the crack growth problems such that

$$
\begin{aligned}
\varepsilon & =\sigma / E \text { for } \sigma \leqslant \sigma_{\mathrm{Y}}, \\
& =\left(\sigma_{\mathrm{Y}} / E\right)\left(\sigma / \sigma_{\mathrm{Y}}\right)^{1 / N} \text { for } \sigma>\sigma_{\mathrm{Y}} .
\end{aligned}
$$

The corresponding hardening rate function is

$$
\begin{aligned}
\frac{1}{h} & =0 \text { for } \Sigma \leqslant \sigma_{\mathrm{Y}}, \\
& =\frac{1}{E}\left[\frac{1}{N}\left(\frac{\Sigma}{\sigma_{\mathrm{Y}}}\right)^{(1-N) / N}-1\right] \text { for } \Sigma>\sigma_{\mathrm{Y}} .
\end{aligned}
$$

\section{NUMERICAL FORMULATION FOR STEADY STATE}

\subsection{Iteration scheme}

The solution scheme for the steady-state small scale yielding problem of Fig. 1 is similar to that developed for conventional flow theories of plasticity by Dean and Hutchinson (1980) and Parks et al. (1981). In outline, it is as follows.

Anticipating a numerical implementation, let $\mathbf{E}$ be a generalized strain vector with components comprised of both the strains and the strain gradients, and let $\boldsymbol{\Sigma}$ be the stress vector containing components of $\sigma$ and $\tau$. Denote the plastic strain vector comprising the components of $\varepsilon^{p}$ and $\eta^{p}$ by $\mathbf{E}^{p}$. The matrix of incremental moduli for plastic loading is denoted by $\mathbf{D}$ such that $\boldsymbol{\Sigma}=\mathbf{D} \dot{\mathbf{E}}$; the corresponding elastic matrix of moduli is denoted by $\mathbf{D}^{\mathrm{e}}$. The solution employs iteration. In each iteration, the core 
procedure is the finite element solution for the displacements, strains and stresses assuming that the plastic strain distribution $\mathbf{E}^{\mathrm{p}}$ is known. In notation standard to the finite element method, let $\mathbf{U}$ be the vector of nodal displacements and let $\mathbf{B}$ be the strain matrix such that $\mathbf{E}=\mathbf{B U}$. The finite element problem for $\mathbf{U}$ in terms of applied boundary forces $\mathbf{F}$ (the remotely applied tractions) and any specified $\mathbf{E}^{p}$ is represented in the standard notation as

$$
\mathbf{K}^{\mathrm{e}} \mathbf{U}=\int_{S} \mathbf{N}^{\mathrm{T}} \mathbf{F} \mathrm{d} S+\int_{V} \mathbf{B}^{\mathrm{T}} \mathbf{D}^{\mathrm{e}} \mathbf{E}^{\mathrm{p}} \mathrm{d} V \quad \text { where } \mathbf{K}^{\mathrm{e}}=\int_{V} \mathbf{B}^{\mathrm{T}} \mathbf{D}^{\mathrm{e}} \mathbf{B} \mathrm{d} V .
$$

The iteration steps are as follows:

(1) Use the distribution of $\mathbf{E}^{\mathrm{p}}$ from the previous iteration in (3.1) to determine $\mathbf{U}$. In the first iteration take $\mathbf{E}^{\mathrm{p}}=\mathbf{0}$.

(2) Compute $\mathbf{E}$ from $\mathbf{U}$.

(3) Obtain a new estimate of the distribution of $\Sigma$. Use $\Sigma=\mathbf{D}^{\mathrm{e}} \mathbf{E}$ in the region where $\Sigma<\sigma_{\mathrm{Y}}$ outside the active plastic zone and outside the wakc. Elscwhere, make use of the fact that for steady-state growth in the $x_{1}$ direction, $\dot{\boldsymbol{\Sigma}}=\mathbf{D} \dot{\mathbf{E}}$ can be replaced by $\partial \mathbf{\Sigma} / \partial x_{1}=\mathbf{D} \partial \mathbf{E} / \partial x_{1}$ such that

$$
\boldsymbol{\Sigma}\left(x_{1}, x_{2}\right)=\boldsymbol{\Sigma}\left(x_{1}^{*}, x_{2}\right)-\int_{x_{1}}^{x_{1}^{*}} \mathbf{D} \partial \mathbf{E} / \partial x_{1} \mathrm{~d} x_{1},
$$

where $\left(x_{1}^{*}, x_{2}\right)$ is any point on the leading edge of the current active plastic zone, i.e. where $\Sigma=\sigma_{\mathrm{Y}}$. The integration in (3.2) is performed for fixed $x_{2}$ and applies to all points to the left of the leading edge.

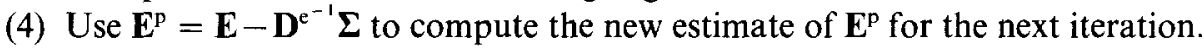

(5) If satisfactory convergence has not been achieved repeat steps (1)-(4).

\subsection{Choice of element}

A discussion of some of the numerical difficulties surrounding the choice of finite element has been given by Xia and Hutchinson (1996) with emphasis on stationary plane strain cracks in the restricted class of CS solids. In the present study, ninenoded isoparametric elements were employed with four Gauss integration points in each element. The mesh was designed to facilitate the integration along streamlines in (3.2).

\subsection{Computation of traction on extended crack plane}

In the higher order theory, the stress quantities $\tau_{i j k}$ contribute to the tractions. The components of the traction vector $t$, the force per unit area given by (2.13), acting on the plane directly ahead of the crack tip, $x_{2}=0$, are

$$
t_{k}=\sigma_{2 k}-2 \tau_{21 k, 1}-\tau_{22 k, 2} .
$$

For mode I fields which are symmetric about the crack plane, $t_{1}=0$ and

$$
t_{2}=\sigma_{22}-2 \tau_{212,1}-\tau_{222,2} \text {. }
$$


The normal traction ahead of the crack tip requires evaluation of the derivative of $\tau_{212}$ and $\tau_{222}$, presenting a particular challenge for accurate numerical computation. An alternative to using (3.4) is obtained by noting from the principle of virtual work (2.10) that $t_{2}$ is work conjugate to $u_{2}$. Thus, $t_{2}$ may be interpreted as a nodal force at nodes along the extended crack plane in the higher order finite element formulation. Both (3.4) and the nodal force from the elements have been used to compute the distribution of normal traction ahead of the crack tip presented in the next section. The two procedures are in good agreement.

\section{TRACTION AND OPENING DISPLACEMENT IN STEADY-STATE GROWTH}

The normal traction $t_{2}$ acting on the plane ahead of the crack tip in the small scale yielding, steady-state problem in Fig. 1 has the same form noted for the stress components in (1.2), i.e.

$$
\frac{t_{2}}{\sigma_{\mathrm{Y}}}=\bar{t}_{2}\left(\frac{x_{1}}{R_{\mathrm{p}}}, \frac{l}{R_{\mathrm{p}}}, \frac{l_{\mathrm{e}}}{l}, N, \frac{\sigma_{\mathrm{Y}}}{E}, v\right),
$$

where $\bar{t}_{2}$ is a dimensionless function of the arguments displayed. The dependence on $l_{\mathrm{e}} / l$ is explicitly listed, although its importance is secondary. The quantity $R_{\mathrm{p}}$ is defined in terms of the remote stress intensity factor $K$ in (1.1). Results for $t_{2} / \sigma_{\mathrm{Y}}$ as a function of $x_{1} / R_{\mathrm{P}}$ for the SG solid are plotted in Fig. 2 for a solid with $l_{\mathrm{e}} / l=0.5, E / \sigma_{\mathrm{Y}}=300$, $v=0.3$ and $N=0.1$ [Fig. 2(a)] and $N=0.2$ [Fig. 2(b)]. In each of the two figures, the curve for $l / R_{\mathrm{P}}=0$ corresponds to the limit for the conventional $\mathrm{J}_{2}$ flow theory solid. The curves for the nonzero values of $l / R_{\mathrm{P}}$ apply to the SG solid. The strong influence of strain gradients as measured by $l / R_{\mathrm{P}}$ in elevating the tractions within a distance of about $R_{\mathrm{P}} / 10$ from the crack tip is evident. Note that at distances further out from the tip, the tractions drop slightly below those for the conventional solid, consistent with the requirement of overall force equilibrium that the higher tractions near the tip be offset by lower values.

The opening displacement of the crack faces behind the crack tip, $\delta=u_{2}\left(x_{1}, 0^{+}\right)-u_{2}\left(x_{1}, 0^{-}\right)$, for the case with $N=0.2$ is plotted in Fig. 3. Strain gradient hardening reduces the opening near the tip, creating a significantly sharper crack tip. It is recalled that the crack tip of a growing crack in the conventional $\mathbf{J}_{2}$ flow theory solid is already quite sharp (Drugan et al., 1982). Boundaries of plastic zones are displayed in Fig. 4 for $l / R_{\mathrm{P}}=0.3$ along with the boundary for the conventional theory $\left(l / R_{\mathrm{P}}=0\right)$. Strain gradient hardening has only a minor effect on the size and shape of the plastic zone. This is not surprising because strain gradients become important only in a small region near the tip, well inside the plastic zone. For $N=0.2$, the halfheight of the steady-state plastic zone is approximately $20 \%$ greater than the reference quantity $R_{\mathrm{P}}$ defined in (1.1). The thin region just above the crack face along the negative $x_{1}$ axis is a zone of plastic reloading.

All the results in Figs 2-4 were computed with $l_{\mathrm{e}} / l$ fixed at 0.5 . The elastic strain gradient contributions were introduced in (2.14) to ensure a positive definite energy 

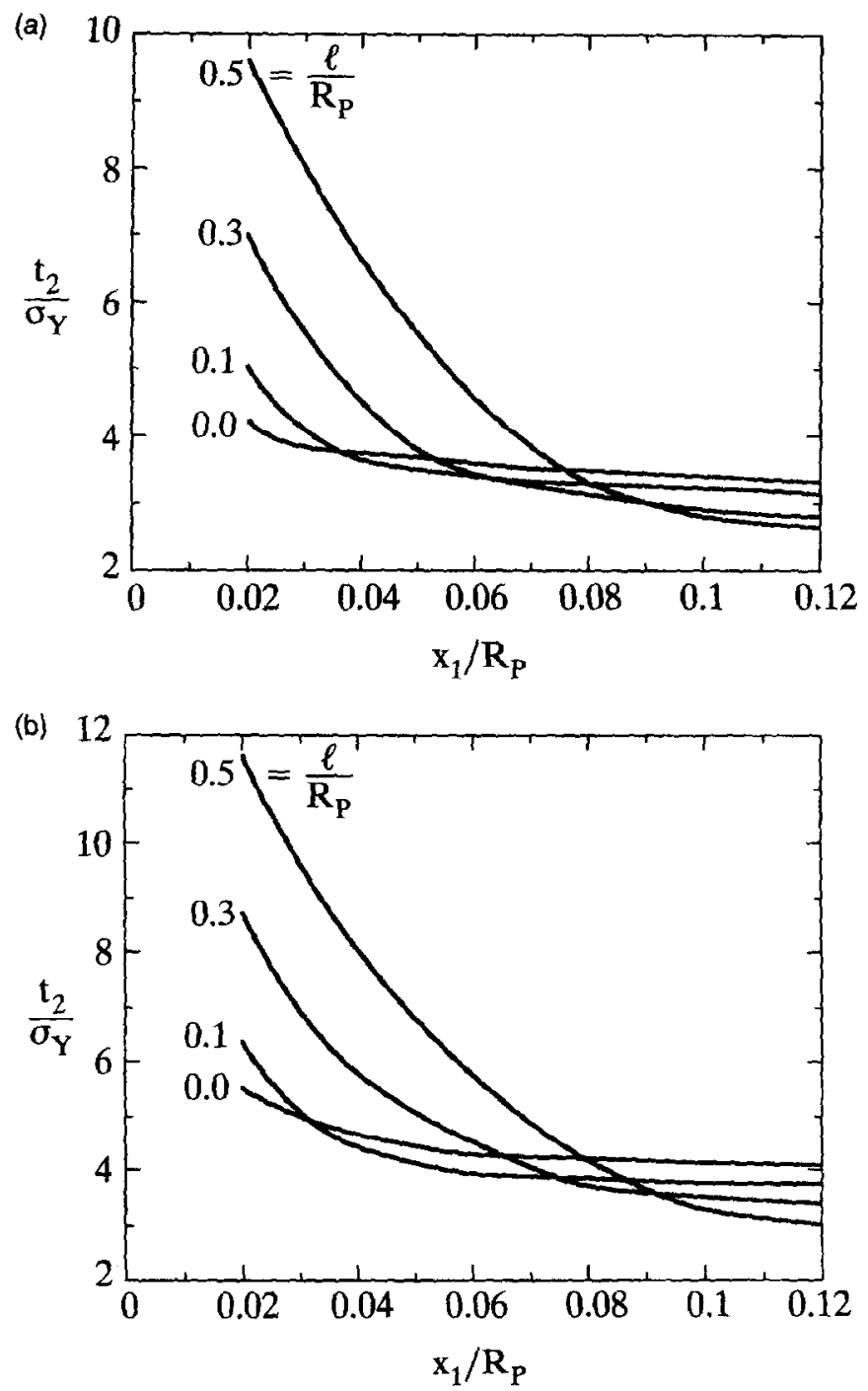

Fig. 2. Traction on the plane ahead of the crack tip in the region where the strain gradients have strong influence. (a) $N=0.1$ and (b) $N=0.2$. The curve for $l / R_{\mathrm{P}}=0$ corresponds to that for conventional $\mathrm{J}_{2}$ flow theory. For all curves, $\sigma_{\gamma} / E=1 / 300, v=0.3$ and $l_{e} / l=0.5$.

density and an invertible constitutive law. They are not considered to have any physical significance for strain gradient plasticity. The relatively minor influence of $l_{\mathrm{e}} / l$ on the traction ahead of the crack tip can be seen in Fig. 5 for the case $N=0.2$.

The distinction between the normal traction acting on the plane ahead of the crack tip, $t_{2}$, and the normal component, $\sigma_{22}$, is illustrated in Fig. 6 , for the cases $l / R_{\mathrm{P}}=0.1$ and 0.3 with $l_{\mathrm{e}} / l=0.5$ and $N=0.2$. The difference, owing to $-2 \tau_{212,1}-\tau_{222,2}$ in (3.4), is all-important in understanding the role of strain gradient hardening in elevating the traction ahead of the tip, i.e. the force per unit area acting on the extended plane 


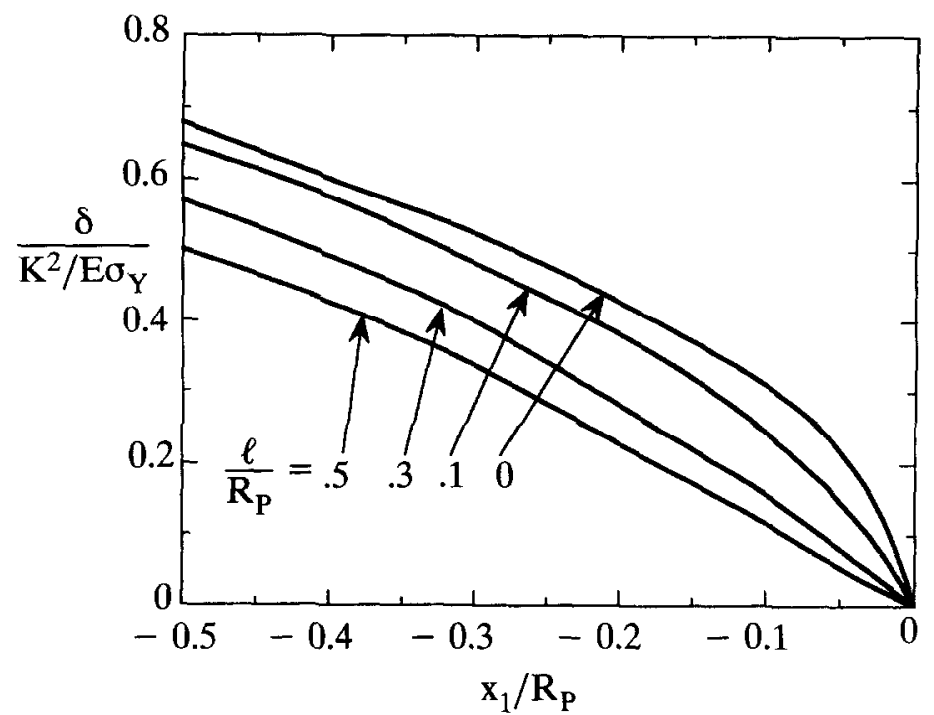

Fig. 3. Normalized crack opening displacement under steady-state growth for various values $l / R_{\mathrm{P}}$ for $N=0.2, \sigma_{\mathrm{Y}} / E=1 / 300, v=0.3$ and $l_{\mathrm{e}} / l=0.5$.

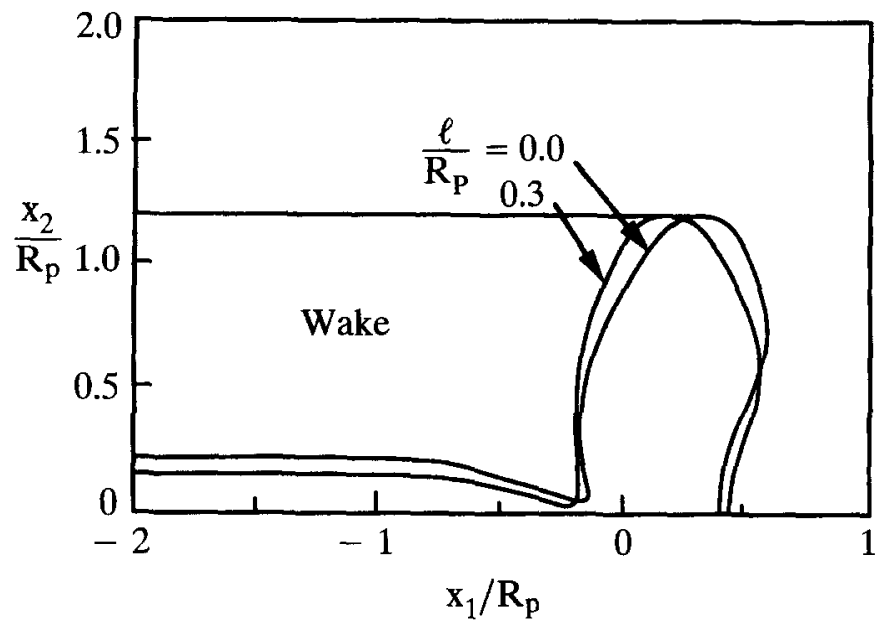

Fig. 4. Active plastic zone and unloading wake for $\mathrm{J}_{2}$ flow theory $\left(l / R_{\mathrm{P}}=0\right)$ and for the $\mathrm{SG}$ solid with $l / R_{\mathrm{P}}=0.3$, in each case with $N=0.2, \sigma_{\mathrm{Y}} / E=1 / 300, v=0.3$ and $l_{\mathrm{e}} / l=0.5$.

of fracture. The component $\sigma_{22}$ is not elevated by gradient effects, and, in fact, is slightly diminished. The larger $l / R_{\mathrm{P}}$ is, the greater difference between $t_{2}$ and $\sigma_{22}$ in the range plotted. The tractions in the above figures have been computed in two ways, as mentioned earlier: using (3.4) with numerical differentiation of $\tau_{212}$ and $\tau_{222}$ (the contribution from $\tau_{222,2}$ is small compared to that from $\tau_{212,1}$ ), and using the nodal forces as interpreted by the Principle of Virtual Work. Values for $t_{2} / \sigma_{\mathrm{Y}}$ computed in these two ways differ only slightly and would be indistinguishable in a plot such as Fig. 6. 


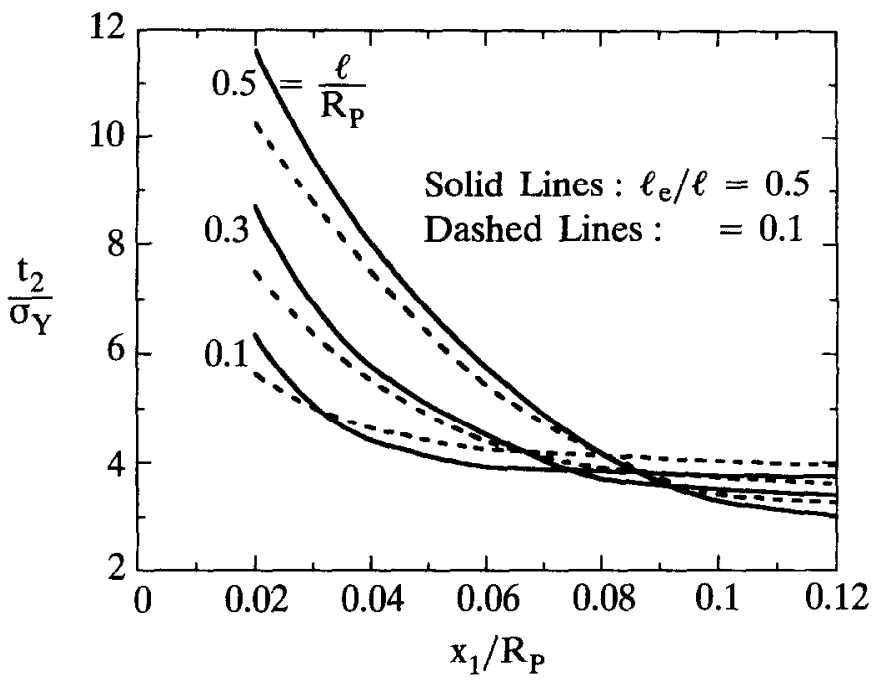

Fig. 5. The effect of $l_{\mathrm{e}} / l$ on traction ahead of the crack tip for several values of $l / R_{\mathrm{P}}$ with $N=0.2$, $\sigma_{\mathrm{Y}} / E=1 / 300$ and $v=0.3$.

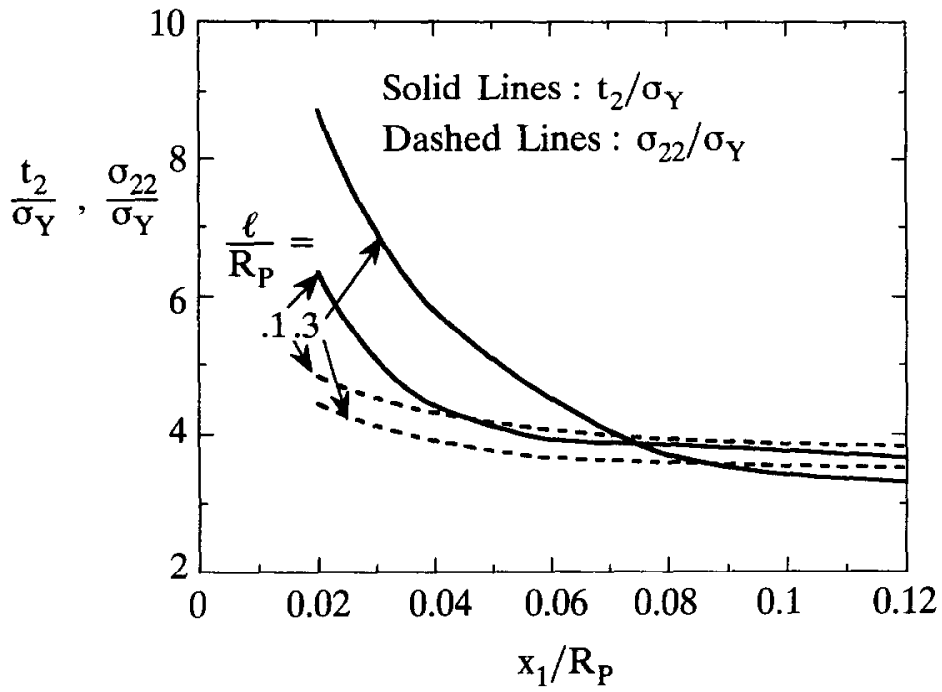

Fig. 6. Traction $t_{2}$ and stress component $\sigma_{22}$ on plane ahead of crack tip for two values of $l / R_{\mathrm{P}}$ with $N=0.2$, $\sigma_{\mathrm{Y}} / E=1 / 300, v=0.3$ and $l_{\mathrm{e}} / l=0.5$.

\section{THE INFLUENCE OF $l$ ON STEADY-STATE FRACTURE TOUGHNESS}

In this section, the SG solid is taken as the description of the elastic-plastic solid in the embedded fracture process zone model (EPZ model) of Needleman (1987) and Tvergaard and Hutchinson (1992). In this model, the traction-separation law characterizing the fracture process is prescribed as a boundary condition along the 

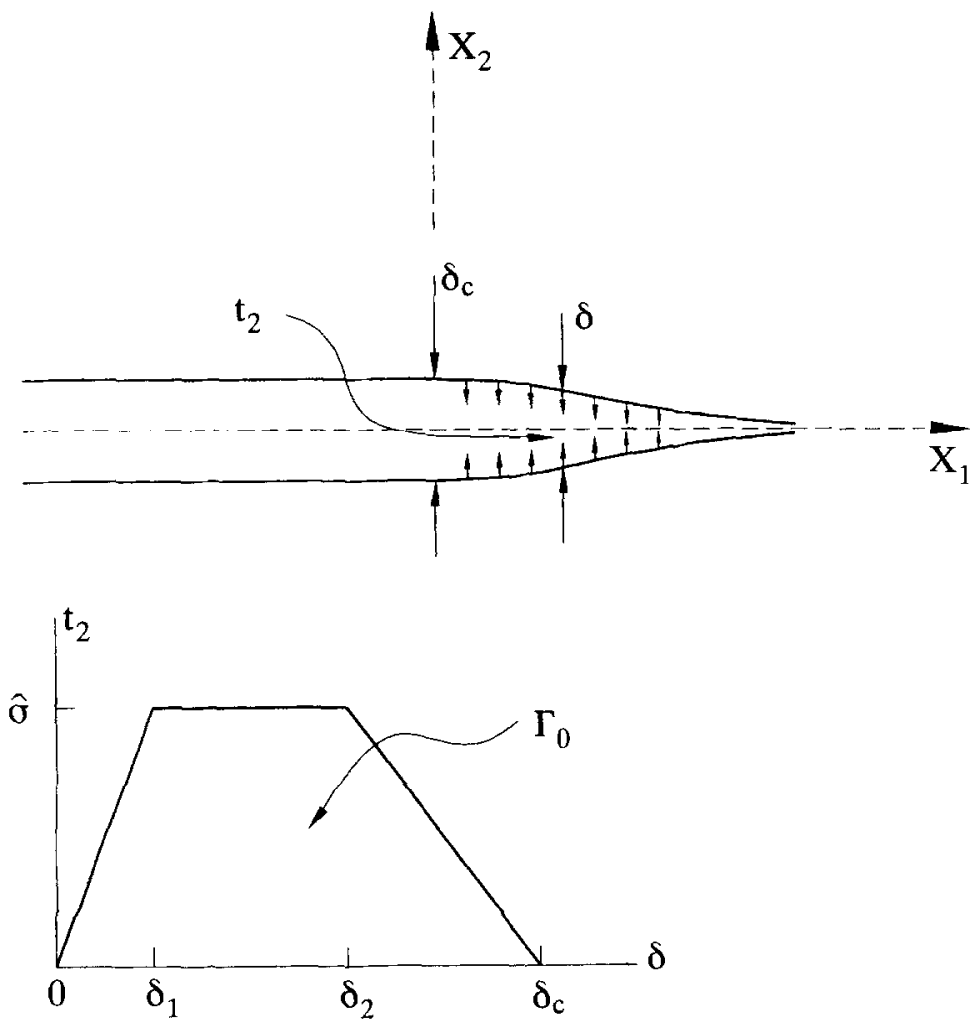

Fig. 7. Embedded process zone model for determination of steady-state toughness. The traction-separation relation characterizing the fracture process is applied along the plane ahead of the tip and is specified by the work of separation $\Gamma_{0}$, the peak traction $\hat{\sigma}$ and the two shape factors $\lambda_{1}=\delta_{1} / \delta_{\mathrm{c}}$ and $\lambda_{2}=\delta_{2} / \delta_{\mathrm{c}}$.

plane ahead of the crack tip, as depicted in Fig. 7. The continuum description of the elastic-plastic solid holds everywhere off the extended fracture plane. Heretofore, conventional $\mathrm{J}_{2}$ flow theory has been used to describe the solid. Attention here will focus on steady-state, mode I plane strain toughness under small scale yielding conditions. Based on the discussion in Section 1 and the results of the previous section, the expectation is for strain gradient hardening to substantially alter the predictions of the EPZ model when the maximum traction required for separation, $\hat{\sigma}$, is large.

The form of the traction-separation law shown in Fig. 7 is exactly the same as that employed in earlier studies (e.g. Tvergaard and Hutchinson, 1992). However, within the context of the higher order theory, it is $t_{2}$, not $\sigma_{22}$, that is work conjugate to the crack opening separation $\delta=u_{2}\left(x_{1}, 0^{+}\right)-u_{2}\left(x_{1}, 0^{-}\right)$. Thus, in the present version of the EPZ model, the relation between $t_{2}$ and $\delta$ in Fig. 7 is prescribed as the condition along the plane ahead of the crack tip (i.e. on $x_{2}=0$ for $x_{1}>0$ ). The work of fracture per unit area, $\Gamma_{0}$, is related to $\hat{\sigma}$ and $\delta$ by

$$
\Gamma_{0}=\int_{0}^{\delta_{\mathrm{c}}} t_{2} \mathrm{~d} \delta=\frac{1}{2} \hat{\sigma} \delta_{\mathrm{c}}\left[1+\lambda_{2}-\lambda_{1}\right],
$$


where $\lambda_{1}=\delta_{1} / \delta_{\mathrm{c}}$ and $\lambda_{2}=\delta_{2} / \delta_{\mathrm{c}}$. Inspection of the principle of virtual work in (2.10) reveals that a contribution from the double strcss component $r_{1}$ working through $u_{1,2}$ along the plane ahead of the crack could also be incorporated into a generalized traction-separation law (a contribution from $r_{2}$ vanishes by symmetry). We have chosen not to include any contribution from $r_{1}$. Because no constraint on $u_{1,2}$ along $x_{2}=0$ is imposed, $r_{1}=0$ is a natural (dynamic) boundary condition enforced by the variational solution process.

The numerical method discussed in Section 3 applies to the steady-state problem for the EPZ model as well as the first problem. Now, however, the iteration scheme must satisfy the traction-separation relation ahead of the tip and must adjust the level of the remote stress intensity $K$ such that the propagation condition at the tip is met, i.e.

$$
\delta=\delta_{\mathrm{c}} \quad \text { at } x_{1}=0 .
$$

The outcome of the calculation is the relation between $K_{\mathrm{ss}}$ and the parameters specifying the fracture process and the SG solid. The results will be presented using the equivalent energetic measure of steady-state toughness

$$
\Gamma_{\mathrm{ss}}=\frac{1-v^{2}}{E} K_{\mathrm{ss}}^{2}
$$

This quantity measures the total, or macroscopic, work of fracture; $\Gamma_{\mathrm{ss}}-\Gamma_{0}$ is the plasticity contribution to the work of fracture. Dimensional considerations now give

$$
\frac{\Gamma_{\mathrm{ss}}}{\Gamma_{0}}=F\left(\frac{l}{R_{0}}, N, \frac{\hat{\sigma}}{\sigma_{\mathrm{Y}}}, \frac{\sigma_{\mathrm{Y}}}{E}, \frac{l_{\mathrm{e}}}{l}, v, \lambda_{1}, \lambda_{2}\right),
$$

where $R_{0}$ is the same reference length used by Tvergaard and Hutchinson (1992):

$$
R_{0}=\frac{1}{3 \pi\left(1-v^{2}\right)} \frac{E \Gamma_{0}}{\sigma_{\mathrm{Y}}^{2}} .
$$

Other than $l$ and $l_{\mathrm{e}}, R_{0}$ is the only length quantity in the model. It can be interpreted as an estimate of the half-height of the plastic zone in the limit that $\Gamma_{\mathrm{ss}}$ is only slightly greater than $\Gamma_{0}$. Equivalently, it can be thought of as the estimate of the half-height of the plastic zone when $K=\sqrt{E \Gamma_{0} /\left(1-v^{2}\right)}$ is applied remotely. Note that $R_{\mathrm{p}}$ defined by (1.1) is precisely $\left(\Gamma_{\mathrm{ss}} / \Gamma_{0}\right) R_{0}$. The nondimensional parameters in (5.4) which have the greatest effect on $\Gamma_{\mathrm{ss}} / \Gamma_{0}$ are $\| / R_{0}, N$ and $\hat{\sigma} / \sigma_{\mathrm{Y}}$. The shape factors for the tractionseparation law, $\lambda_{1}$ and $\lambda_{2}$, have been shown from the earlier study to be relatively unimportant. From (5.1) it can be noted that the critical separation $\delta_{\mathrm{c}}$ is determined when $\Gamma_{0}, \hat{\sigma}$ and the shape factors have been specified.

The role of strain gradient hardening in determining toughness is seen in Fig. 8 for the case of a solid with moderately high strain hardening, $N=0.2$. The limiting curve for $l / R_{0}=0$ is that for the conventional $\mathrm{J}_{2}$ flow theory solid, which is in agreement with the result obtained by Tvergaard and Hutchinson (1992) for the same model. The effect of replacing the conventional solid by the SG solid in the model is profound. Strain gradient hardening elevates the traction ahead of the crack tip, thereby allowing higher peak separation stresses to be overcome. The trends displayed in Fig. 8 are 


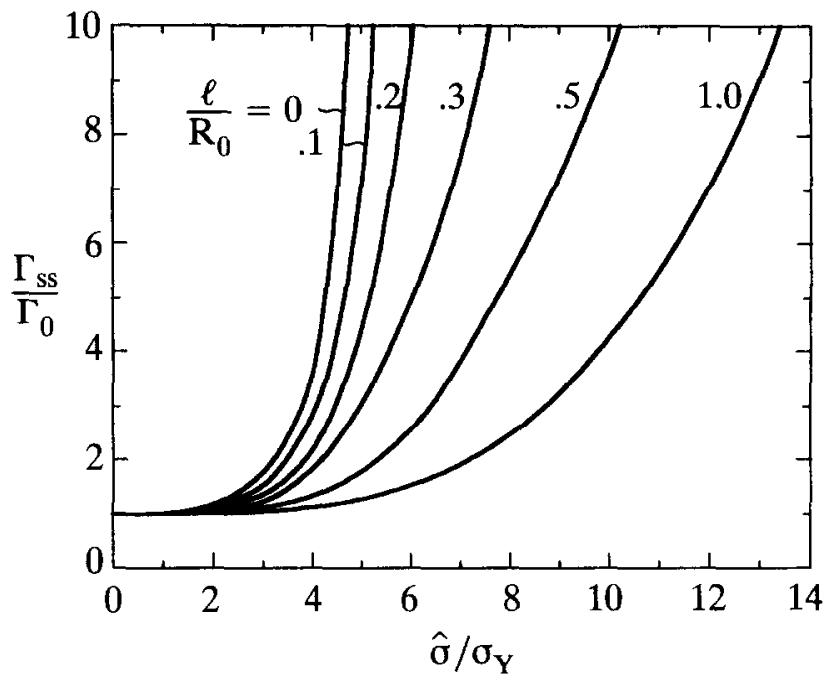

Fig. 8. Influence of strain gradient effects on steady-state toughness. Curves of $\Gamma_{\text {ss }} / \Gamma_{0}$ as a function of the normalized peak separation traction, $\hat{\sigma} / \sigma_{\mathrm{Y}}$, for various values of $l / R_{0}$ with $N=0.2, \sigma_{\mathrm{Y}} / E=1 / 300, v=0.3$, $\lambda_{1}=0.15, \lambda_{2}=0.5$ and $l_{\mathrm{e}} / l=0.5$. The curve for $l / R_{0}=0$ agrees with that obtained by Tvergaard and

Hutchinson (1992) for the conventional $\mathbf{J}_{2}$ flow theory solid.

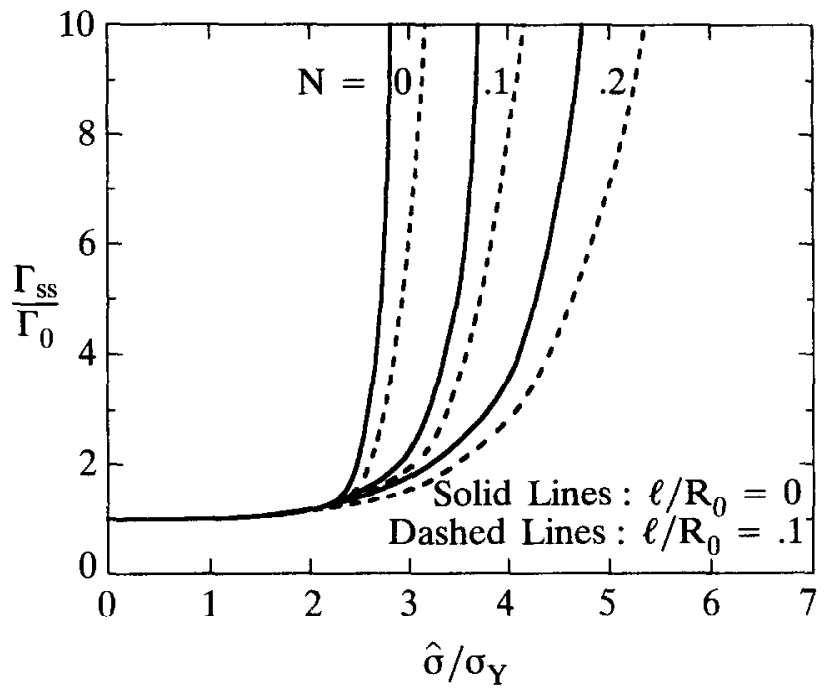

Fig. 9. Influence of a small value of $l / R_{0}$ on the relation of $\Gamma_{\mathrm{ss}} / \Gamma_{0}$ to $\hat{\sigma} / \sigma_{\mathrm{Y}}$ for several levels of strain hardening $N$. The curves for $l / k_{0}=0$ are in agreement with those obtained by Tvergaard and Hutchinson (1992) for the conventional $\mathrm{J}_{2}$ flow theory solid. $\left(\sigma_{\mathrm{Y}} / E=1 / 300, v=0.3, \lambda_{1}=0.15, \lambda_{2}=0.5\right.$ and $l_{\mathrm{e}} / l=0.5$.)

seen at all levels of strain hardening. Figure 9 shows the effect of a relatively small value of $l / R_{0}$ on the steady-state toughness for three levels of strain hardening. The effect is greatest at the highest level of strain hardening, but it persists even for the case with $N=0$. 


\section{CONCLUDING REMARKS}

The trends in Fig. 8 appear to go a long way towards overcoming the limitations of the EPZ model discussed in Section 1 . Specifically, with values of $l / R_{0}$ larger than about $1 / 2$, peak separation tractions well above $10 \sigma_{\mathrm{Y}}$ can be attained. Alomic separation of a metal lattice or a metal/ceramic interface typically requires a work of separation, $\Gamma_{0}$, on the order of several $\mathrm{J} \mathrm{m}^{-2}$. Using representative values for $E$ and $\sigma_{\mathrm{Y}}$ in (5.5), one finds values of $R_{0}$ in the range from about 0.1 to $1 \mu \mathrm{m}$. Given that experimental data indicate that $l$ is likely to be on the order of a micrometer or more [4 $\mu \mathrm{m}$ for annealed copper; Fleck et al. (1994)], values of $l / R_{0}$ at least as large as unity must be expected for fracture processes based on separation at the atomic scale. By contrast, when the fracture process is void nucleation, growth and coalescence ahead of the crack tip, the work of the fracture process is approximately $\Gamma_{0}=\sigma_{\mathrm{Y}} D / 2$, where $D$ is the average void spacing (Tvergaard and Hutchinson, 1992). For the void mechanism, typical values of $\Gamma_{0}$ are of the order of $1 \mathrm{KJ} \mathrm{m}^{-2}, R_{0}$ is a fraction of a $\mathrm{mm}$ or more and $l / R_{0}$ is expected to be so small that strain gradient effects will have little influence on toughness predictions.

Plastic strain gradient effects similar to those just described are likely to be comparably important for the Suo, Shih and Varias (SSV) model (Suo et al., 1993; Beltz et al., 1996). The width $D$ of the elastic region at the crack tip from which plastic deformation is excluded in the SSV model is typically on the order of a small fraction of a micron when the model is applied to fracture governed by an atomic separation mechanism. It is most unlikely that conventional plasticity theory can be used down to that scale to model plastic deformation outside the elastic exclusionary zone. It seems reasonable to expect that the tractions acting on the elastic strip region will attain significantly higher levels than predicted by conventional plasticity.

\section{ACKNOWLEDGEMENTS}

This work was supported in part by the Office of Naval Research under Grant N00014-960559, by the National Science Foundation under Grant CMS-9634632, and by the Division of Applied Sciences, Harvard University.

\section{REFFRENCES}

Bagchi, A. and Evans, A. G. (1996) The mechanics and physics of thin film decohesion and its measurement. Interface Sci. 3, 169-193.

Bagchi, A., Lucas, G. E., Suo, Z. and Evans, A. G. (1994) A new procedure for measuring the decohesion energy of thin ductile films on substrates. J. Mater. Res. 9, 1734-1741.

Beltz, G. E., Rice, J. R., Shih, C. F. and Xia, L. (1996) A self-consistent model for cleavage in the presence of the plastic flow. Acta Mater. 44, 3943-3954.

Dean, R. H. and Hutchinson, J. W. (1980) Quasi-static steady crack growth in small scale yielding. In Fracture Mechanics, ASTM STP 700, pp. 383-405. American Society for Testing Materials.

Drugan, W. J., Rice, J. R. and Sham, T.-L. (1982) Asymptotic analysis of growing plane strain tensile cracks in elastic-ideally plastic solids. J. Mech. Phys. Solids 30, 447-473. 
Elssner, G., Korn, D. and Rühle, M. (1994) The influence of interface impurities on fracture energy of UHV diffusion bonded metal-ceramic bicrystals. Scripta Metall. Mater. 31, 1037 1042.

Fleck, N. A. and Hutchinson, J. W. (1997) Strain gradient plasticity. Advances in Applied Mechanics, Vol. 33, ed. J. W. Hutchinson and T. Y. Wu, pp. 295-361.

Fleck, N. A., Muller, G. M., Ashby, M. F. and Hutchinson, J. W. (1994) Strain gradient plasticity: theory and experiment. Acta Metall. Mater. 42, 475-487.

Freund, L. B. and Hutchinson, J. W. (1985) High strain-rate crack growth in rate-dependent plastic solids. J. Mech. Phys. Solids 33, 169-191.

Huang, Y., Zhang, L., Guo, T. F. and Hwang, K. C. (1997) Near-tip fields for cracks in materials with strain gradient effects. In Proc. IUTSM Symp. on Nonlinear Analysis of Fracture, ed. J. W. Willis. Pp. 231-244. Kluwer Academic Publishers, Dordrecht.

Ma, Q. and Clarke, D. R. (1995) Size dependent hardness of silver single crystals. J. Mater. Res. 10(4), 853-863.

Mindlin, R. D. (1964) Micro-structure in linear elasticity. Arch. Ration. Mech. Anal. 16, 5178.

Needleman, A. (1987) A continuum model for void nucleation by inclusion debonding. $J$. Appl. Mech. 54, 525-531.

Parks, D. M., Lam, P. S. and McMeeking, R. M. (1981) Some effects of inelastic constitutive models on crack tip fields in steady crack growth. Advances in Fracture Research, ed. D. Francois, Vol. 5, pp. 2607-2614. Pergamon Press.

Poole, W. J., Ashby, M. F. and Fleck, N. A. (1996) Micro-hardness of annealed and workhardened copper polycrystals. Scripta Metall. Mater. 34(4), 559-564.

Schiermeier, A. D. and Hutchinson, J. W. (1996) Stationary crack tip fields in mode I for strain gradient plasticity, in preparation.

Schiermeier, A. D., Huang, Y., Hutchinson, J. W. and Hwang, K. C. (1996) Stationary crack tip fields in mode III for strain gradient plasticity, in preparation.

Smyshlyaev, V. P. and Fleck, N. A. (1996) The role of strain gradients in the grain size effect for polycrystals. J. Mech. Phys. Solids 44, 465-496.

Stelmashenko, N. A., Walls, M. G., Brown, L. M. and Milman, Y. V. (1993) Microindentations on W and Mo oriented single crystals: an STM study. Acta Metall. Mater. 41(10), 28552865.

Suo, Z., Shih, C. F. and Varias, A. G. (1993) A theory for cleavage cracking in the presence of plastic flow. Acta Metall. Mater. 41, 151-1557.

Toupin, R. A. (1962) Elastic materials with couple stresses. Arch. Ration. Mech. Anal. 11, 385414.

Tvergaard, V. and Hutchinson, J. W. (1992) The relation between crack growth resistance and fracture process parameters in elastic-plastic solids. J. Mech. Phys. Solids 40, 1377-1397.

Xia, Z. C. and Hutchinson, J. W. (1996) Crack tip fields in strain gradient plasticity $J$. Mech. Phys. Solids 44, 1621-1648.

\section{APPENDIX}

Orthogonal decomposition and projection tensors

Construction of the Smyshlyaev-Fleck decomposition (2.2) of $\eta_{i j k}=\eta_{i j k}$ starts with the formation of $\eta_{i j k}^{(4)} \equiv \eta_{i j k}^{\mathrm{H}}$ and $\eta_{i j k}^{\prime}=\eta_{i j k}-\eta_{i j k}^{\mathrm{H}}$ as in Section 2.1. A fully symmetric tensor $\eta^{\mathrm{S}}$ is formed according to

$$
\eta_{i j k}^{\mathrm{S}} \equiv \frac{1}{3}\left[\eta_{i j k}^{\prime}+\eta_{j k i}^{\prime}+\eta_{k i j}^{\prime}\right]
$$

Then,

$$
\eta_{i j k}^{(1)}=\eta_{i j k}^{\mathrm{s}}-\frac{1}{5}\left[\delta_{i j} \eta_{k \mathrm{pp}}^{\mathrm{s}}+\delta_{j k} \eta_{i \mathrm{pp}}^{\mathrm{s}}+\delta_{k i} \eta_{j \mathrm{pp}}^{\mathrm{s}}\right]
$$




$$
\begin{gathered}
\eta_{i j k}^{(2)}=\frac{1}{6}\left[e_{i k \mathrm{p}} e_{j l m} \eta_{l \mathrm{p} m}^{\prime}+e_{j k p} e_{i l m} \eta_{l \mathrm{p} m}^{\prime}+2 \eta_{i j k}^{\prime}-\eta_{j k i}^{\prime}-\eta_{k i j}^{\prime}\right] \\
\eta_{i j k}^{(3)}=\frac{1}{6}\left[-e_{i k \mathrm{p}} e_{j l m} \eta_{l \mathrm{p} m}^{\prime}-e_{j k \mathrm{p}} e_{i l m} \eta_{l \mathrm{p} m}^{\prime}+2 \eta_{i j k}^{\prime}-\eta_{j k i}^{\prime}-\eta_{k i j}^{\prime}\right]+\frac{1}{5}\left[\delta_{i j} \eta_{k \mathrm{pp}}^{\mathrm{s}}+\delta_{j k} \eta_{\mathrm{pp}}^{\mathrm{s}}+\delta_{k i} \eta_{j \mathrm{pp}}^{\mathrm{s}}\right]
\end{gathered}
$$

The associated projection tensors defined in (2.17) are given by

$$
\begin{gathered}
T_{i j k p q r}^{(n)}=\frac{1}{6}\left\{\left(\delta_{i p} \delta_{j q}+\delta_{i q} \delta_{j p}\right) \delta_{k r}+\left(\delta_{j p} \delta_{k q}+\delta_{j q} \delta_{k p}\right) \delta_{i r}+\left(\delta_{i p} \delta_{k q}+\delta_{i q} \delta_{k p}\right) \delta_{j r}\right\} \\
-\frac{1}{15}\left\{\left(\delta_{i j} \delta_{k r}+\delta_{j k} \delta_{i r}+\delta_{k i} \delta_{j r}\right) \delta_{p q}+\left(\delta_{i j} \delta_{k p}+\delta_{j k} \delta_{i p}+\delta_{k i} \delta_{j p}\right) \delta_{q r}+\left(\delta_{i j} \delta_{k q}+\delta_{j k} \delta_{i q}+\delta_{k i} \delta_{j q}\right) \delta_{r p}\right\} \\
\begin{array}{r}
T_{i j k p q r}^{(2)}=\frac{1}{12}\left\{e_{i k q} e_{j p r}+e_{j k q} e_{i p r}+e_{i k p} e_{j q r}+e_{j k p} e_{i q r}\right\} \\
\quad+\frac{1}{12}\left\{2\left(\delta_{i p} \delta_{j q}+\delta_{i q} \delta_{j p}\right) \delta_{k r}-\left(\delta_{j p} \delta_{k q}+\delta_{j q} \delta_{k p}\right) \delta_{i r}-\left(\delta_{k p} \delta_{i q}+\delta_{k q} \delta_{i p}\right) \delta_{j r}\right\} \\
T_{i j k p q r}^{(3)}=\frac{1}{12}\left\{-e_{i k q} e_{j p r}-e_{j k q} e_{i p r}-e_{i k p} e_{j q r}-e_{j k p} e_{i q r}\right\}-\frac{1}{8}\left\{\left(\delta_{i p} \delta_{j k}+\delta_{j p} \delta_{i k}\right) \delta_{q r}+\left(\delta_{i q} \delta_{j k}+\delta_{j q} \delta_{i k}\right) \delta_{p r}\right\} \\
\quad+\frac{1}{12}\left\{2\left(\delta_{i p} \delta_{j q}+\delta_{i q} \delta_{j p}\right) \delta_{k r}-\left(\delta_{j p} \delta_{k q}+\delta_{j q} \delta_{k p}\right) \delta_{i r}-\left(\delta_{k p} \delta_{i q}+\delta_{k q} \delta_{i p}\right) \delta_{j r}\right\} \\
+\frac{1}{15}\left\{\left(\delta_{i j} \delta_{k r}+\delta_{j k} \delta_{i r}+\delta_{k i} \delta_{j r}\right) \delta_{p q}+\left(\delta_{i j} \delta_{k p}+\delta_{j k} \delta_{i p}+\delta_{k i} \delta_{j p}\right) \delta_{q r}+\left(\delta_{i j} \delta_{k q}+\delta_{j k} \delta_{i q}+\delta_{k i} \delta_{j q}\right) \delta_{r p}\right\} \\
T_{i j k p q r}^{(4)}=\frac{1}{8}\left\{\left(\delta_{i k} \delta_{j p}+\delta_{j k} \delta_{i p}\right) \delta_{q r}+\left(\delta_{i k} \delta_{j q}+\delta_{j k} \delta_{i q}\right) \delta_{p r}\right\} .
\end{array}
\end{gathered}
$$

Inversion of (2.24)

Write $(2.24)$ as

$$
\begin{aligned}
\dot{\varepsilon}_{i j} & =A_{i j k l} \dot{\sigma}_{k l}+B_{i j l m n} \dot{t}_{l m n} \\
\dot{\eta}_{i j k} & =B_{i j k l m} \dot{\sigma}_{l m}+C_{i j k l m n} \dot{t}_{l m n}
\end{aligned}
$$

and write its inverse as

$$
\begin{aligned}
\dot{\sigma}_{i j} & =D_{i j k l} \dot{\varepsilon}_{k l}+E_{i j l m n} \dot{\eta}_{l m n} \\
\dot{\tau}_{i j k} & =E_{i j k l m} \dot{\varepsilon}_{i m}+F_{i j k l m n} \dot{\eta}_{l m n} .
\end{aligned}
$$

Substitution of (A.10) into (A.9) gives

$$
\begin{aligned}
A_{i j k l} D_{k l p q}+B_{i j k l m} E_{k l m p q} & =I_{i j p q} \\
A_{i j k l} E_{k l p q r}+B_{i j k l m} F_{k l m p q r} & =0 \\
B_{i j k l m} D_{l m p q}+C_{i j k l m n} E_{l m n p q} & =0 \\
B_{i j k l m} E_{i m p q r}+C_{i j k l m m} F_{l m n p q r} & =I_{i j k p q r}
\end{aligned}
$$

where the fourth and sixth order identity tensors have been introduced. The third and fourth of the above equations give

$$
\begin{aligned}
E_{l m n p q} & =-C_{l m n i j k}^{-1} B_{i j k r s} D_{r s p q} \\
F_{\text {lmnpqr }} & =C_{\text {lmnpqr }}^{-1}-C_{l m n i j k}^{-1} B_{i j k r s} E_{r s p q r}
\end{aligned}
$$

where $C^{-1}$ is the inverse of $C$. Elimination of $E$ by combining the first of the equations in (A.11) and (A.12) gives

$$
\left[A_{i j k l}-B_{i j a b c} C_{a b c r s t}^{-1} B_{r s t k l}\right] D_{k l p q}=I_{i j p q}
$$

The key step in the derivation is the determination of $C^{-1}$. This inversion is facilitated by the properties of the projection tensors in (2.17) and (2.18) and the fact that the $\tau^{(I)}$ are orthogonal to one another. Anticipate that $C^{-1}$ has a similar form to $C$ defined in (2.24), i.e. 


$$
C_{i j k r s t}^{-1}=J_{i j k r s t}+d \sum_{I=1}^{3} \alpha^{(n)} \tau_{i j k}^{(I)} \sum_{J=1}^{3} \alpha^{(n)} \tau_{r s t}^{(j)}
$$

where $d$ and $\alpha^{(n)}(I=1,3)$ are to be determined. Direct substitution of (A.14) into $C^{-1} C=I$ reveals that (A.14) is valid if

$$
\begin{aligned}
d & =-8(1+v) E\left(l_{\mathrm{e}} / l\right)^{4} /\left[H \sigma_{\mathrm{e}}^{2}\right] \\
\alpha^{(I)} & =\left(\kappa_{\mathrm{e}}^{(I)} / \kappa^{(I)}\right)^{2}
\end{aligned}
$$

where $H$ is defined in (2.26). It remains to solve sequentially for $D$ from (A.13), for $E$ from the first equation in (A.12), and, lastly, for $F$ from the second equation in (A.12). The resulting tensors are given in (2.25). 